\title{
UM ESTUdo BibliomÉTRICO do CONGRESSO BRASILEIRO DE CUSTOS NO LAPSO TEMPORAL DE 2011 A 2013
}

\author{
A STUDY OF BIBLIOMETRIC BRAZILIAN CONGRESS OF COSTS IN TIME LAPSE OF 2011 TO 2013
}

\author{
Geovane Camilo Santos \\ geovane_camilo@yahoo.com.br \\ Centro Universitário de Patos de Minas
}

\author{
Laise Teixeira Silva \\ laiseteixeirasilva@hotmail.com
}

\begin{abstract}
RESUMO
A presente pesquisa visou apresentar o perfil dos artigos aprovados para publicação nos anais do Congresso Brasileiro de Custos, entre os anos de 2011 a 2013, sendo utilizado a bibliometria.Os principais pontos abordados foram: número de trabalhos aprovados, gêneros de autores, autores mais prolíferos, faculdades mais prolíferas, abordagem mais usada e maior número de palavras-chave. A metodologia do presente trabalho foi bibliográfica e documental para os procedimentos, descritiva para objetivo e qualitativa e quantitativa para a abordagem. Os resultados apontam que o número de artigos publicados no lapso temporal não apresenta uniformidade, constatou-se a predominância de autores do sexo masculino em relação ao feminino e que os autores mais prolíferos foram: Alceu Souza, Altair Borgert, Carlos Eduardo Facin Lavarda, Fabrícia Silva da Rosa, Ilse Maria Beuren e Sérgio Murilo Petri, com 8 artigos cada um. A faculdade mais prolífera foi a UFSC com 206 publicações e no trato da metodologia o mais usual é não mencionar a abordagem. Na análise do número de palavras-chave verificou que o mais usual são 3 correspondendo a $83,41 \%$ dos artigos.
\end{abstract}

Palavras-chave: Congresso Brasileiro de Custos. Temáticas. Bibliometria.

\begin{abstract}
This study research present aimed to a profile of articles accepted for publication in the Annals of the Brazilian Congress of costs between the years 2011-2013, being used bibliometrics. The main points raised were: number of approved works, genres, authors, most prolific authors, most prolific colleges, most used approach and greater number of keywords. The methodology of this study was to bibliographic and documentary procedures, for objective and descriptive qualitative and quantitative approach to. The results show that the number of published articles in the time gap shows no uniformity, there was a predominance of male authors than female and that the most prolific authors were: Alcaeus Souza, Altair Borgert, Carlos Eduardo Facin Lavarda, Fabrícia Silva Rosa, Ilse Maria Beuren and Sergio Murilo Petri with 8 items each. The most prolific college was UFSC with 206 publications and in dealing with the more usual approach is not to mention the approach. In the analysis of the number of keywords found that the most common are 3 corresponding to $83.41 \%$ of the articles.
\end{abstract}

Keywords: Brazilian Congress of Costs. Thematic. Bibliometrics. 


\section{INTRODUÇÃo}

Desde quando Deus criou o ser humano, este tem o desejo insaciável pelo conhecimento, segundo Maturana e Varela (1995) o criar do mundo foi o cerne pulsante do conhecimento, estando associado às raízes do ser cognitivo.

Quando o ser humano usa a inteligência, primeiramente demonstra a linguagem e após desenvolve os movimentos. O homem segue sempre em busca de mais aprendizado, sendo que se verificam duas formas fundamentais de adquiri-lo; o primeiro é pertencer a um grupo, pois o indivíduo trás consigo o que ele aprendeu no ambiente que ele viveu e vive (VIAUD, 1964) e a segunda forma é por meio da leitura, e quando se a faz, é possível escrever.

A escrita é usada pelo homem desde os tempos primórdios, sendo que inicialmente era por meio de desenho e com o passar do tempo foi desenvolvendo até torna-se escrita como a usual.

Uma maneira admirável de melhorar a forma de escrever dos autores para revistas é escrever artigos para congressos. Ao escreve para estes eventos, além dos artigos serem avaliadospara serem aprovados ou não para a apresentação, eles também serão debatidos no momento da apresentação.

Segundo Slomskiet al (2013) a produção de conhecimento é eficaz, quando a qualidade formal retira todo o seu potencial transformador. Várias vezes os pesquisadores são chamados a julgar a qualidade da pesquisa produzida na área do domínio do seu conhecimento. As maneiras mais eficazes dele demonstrar seu conhecimento são avaliando artigos submetidosa revistas ou a congressos científicos ou participando de bancas de dissertações de mestrado ou teses de doutorado.

Para Larocca, Rosso e Souza (2005) para que a qualidade seja formal e tenha progresso do conhecimento científico dois aspectos são precípuos, avaliação constante e comunicação da produção científica.

Segundo Slomskiet al (2013) os estudos que tratam de análises da produção científica são usados em todas as áreas do conhecimento. Os estudos têm a ideia de que é importante os pesquisadores estarem atentos para a natureza do conhecimento gerado nos seus estudos, assim como, os fundamentos que direcionam as investigações.

Uma das maneiras mais comuns de se realizar pesquisas com foco na análise da produção científica são os estudos bibliométricos. Para Meloet al (2012a) estes estudos analisam o desenvolvimento e difusão de pesquisas científicas, além de aglomerar informações sobre as publicações escritas sobre um tema tratados pelas produções científicas. Segundo Moraeset al (2013) estas pesquisas permitem sustentação para elaboração de novos trabalhos.

Os trabalhos bibliométricos não é algo novo e vem constantemente sendo publicados em eventos e periódicos científicos no Brasil. (MORAES ET AL, 2013).

Existem várias possibilidades de temas para os autores escolherem e escreverem, tanto para congressos quanto para revistas,um destes temas é os estudos bibliométricos.

$O$ presente trabalho tem como problema verificar o perfil dos artigos publicados no Congresso Brasileiro de Contabilidade, no período de 2011 a 2013?

O objetivo geral deste artigo é realizar um estudo bibliométrico dos artigos aprovados para publicação nos anais do CongressoBrasileirode Custo, nos 3últimos anos.

Os principais pontos avaliados no presente trabalho são: (i) números de artigos, (ii) gêneros dos autores, (iii) autores mais prolíferos, (iv) instituição de ensino mais prolífera, (v) metodologia e (vi) quantidade de palavras-chave.

O CBC nos 3 últimos anos teve 7 temáticas, sendo elas: Metodologias de ensino e pesquisa em custos (MEPC), Custos como ferramenta para o planejamento, controle e apoio a decisão (CFPCAD), Métodos quantitativos aplicados a gestão de custos (MQAGC), Custos aplicados ao setor privado e terceiro setor (CASPTS), Custos aplicados ao setor público(CASP),

R. Cont. Ufba, Salvador-Ba, v. 9, n. 1, p. 58 - 72, jan-mar 2015 
Contribuições teóricas para a determinação e a gestão de custos (CTDGC) e Abordagens contemporâneas de custos (ACC). (CBC, 2013a).

O CBC é um dos congressos mais importantes do setor contábil brasileiro, pois além de tratar de variados temas que envolvem a Contabilidade, ele trata especificamente de custos, que é uma das ramificações mais relevantes desta ciência, pois é por meio dele que são possíveis as entidades e pessoas controlarem seus gastos e aumentarem os seus resultados.

O Congresso Brasileiro de Custos trata da importância da aplicação dos custos em 4 setores distintos da sociedade.

O primeiro é o meio acadêmico, com as temáticas: MEPC, MQAGC, CTDGC e ACC. A aplicação neste meio é fundamental, pois ele é a base para o futuro profissional direcionar a sua carreira, pois assim, saberá o que lhe causará grandes oportunidades e como deverá ser sua vida em relação aos custos.

O segundo trato é para a Contabilidade do terceiro setor com a temática: CASPTS, sendo esta abordagem precípua, uma vez, que o terceiro setor é voltado a gerar serviços de caráter público e logo o estudo de custo neste setor ajudará a melhorar a condição de vida de pessoas que atuam direta ou indiretamente nesta área.

O terceiro trato é a ramificação pública da ciência contábil, sendo esta de suma importância ao homem, uma vez que ajudará os responsáveis pela gestão governamental a tomarem as suas decisões, e se possuem um controle de custos terá melhores oportunidades de tomarem decisões voltadas à população. A temática que o CBC dedicou a esta área é CASP.

E o setor empresarial tem para si a temática CFPCAD, o estudo de custos neste setor torna imprescindível, pois as empresas são as principais geradoras de serviços a população, e para que a empresa possa continuar no mercado e "alimentando várias bocas" ela precisa de um estudo de custos com alto grau de qualidade.

Logo, o $\mathrm{CBC}$ e as suas temáticas são importantes à população, pois iniciará com o aperfeiçoamento de alunos, passando pelo terceiro setor, transcorrendo pelo setor público e chegando no setor empresarial.

Neste sentido, o estudo bibliométrico permite uma melhor apresentação da importância desses dados para a Contabilidade, uma vez que abordará as principais áreas que o ser humano está envolvido.

\section{REFERENCIAL TEÓRICO}

Nesta seção apresenta-se o referencial teórico, que melhor situa o leitor sobre o tema proposto. Os assuntos definidos nesta seção foram conceitos de estudo bibliométrico, as leis da bibliometria e do Congresso Brasileiro de Custos.

\subsection{ESTUDO BIBLIOMÉTRICO}

A palavra bibliometria provém da fusão do sufixo "metria" com bibliografia, informação, ciência e biblioteca, respectivamente e são análogos com sua natureza, objetivos e aplicações. (SILVAET AL, 2012). Então, a bibliometria é o estudo que analisa os textos científicos de alguma área específica da produção já pesquisada.

O surgimento da bibliometria remonta ao início do século XX e trabalha como "ferramenta" para acompanhar o crescimento e desenvolvimento das áreas de pesquisa. (SILVA ET AL, 2012).

Segundo Melo et al (2012a) os estudos bibliométricos aumentaram, proporcionando aumento da busca de análises desenvolvidas e difundidas das pesquisas científicas.

R. Cont. Ufba, Salvador-Ba, v. 9, n. 1, p. 58 - 72, jan-mar 2015 
Em conformidade a Oliveiraet al (2013) a bibliometria é precípua para difundir a produção científica, sendo que seu objetivo é alcançado, por meio de uma técnica, que mede a influência dos pesquisadores ou periódicos, permitindo assim, traçar o perfil dos mesmos e as tendências e permite evidenciar áreas temáticas.

Segundo Lima, Diniz e Silva (2012) a bibliometria é uma pesquisa que emprega metodologia estatística, sendo sua aplicação encontrada nas mais variadas publicações. Estas metodologias referem à mensuração de variáveis, como por exemplo, fonte utilizada para produção, gênero autores, instituição e autores mais prolíferos, entre outros.

De acordo com Monteiroet al (2011) estes estudos identificam características e categorias de publicações sobre um assunto, com a finalidade de verificar o perfil das pesquisas que foram desenvolvidas em um lapso temporal.

\subsection{LEIS DA BIBLIOMETRIA}

Na realização dos estudos bibliométricos, para que o trabalho seja elaborado da forma mais segura e mais clara existem leis que orientam estes estudos, sendo elas: Lei de Bradford, Lei de Lotka eLei de Zipf.

Segundo Vanti (2002) a Lei de Bradford trata da dispersão da literatura periódica científica, ou seja, está relacionada com a produtividade de periódicos. (grifo nosso).

Ela determina que quando surgem novos assuntos escritos, estes devam ser submetidos a uma pequena filtragem, por periódico, relacionados, quando aceitos, sendo que estes periódicos atrairão mais artigos (SILVA ET AL, 2012), ou seja, ela mensura a atração de periódicos, principalmente, a assuntos considerados novos. (RIBEIRO, 2013).

A Lei de Lotka ou Lei dos quadrados inversos estuda a produtividade dos autores e determina que aqueles com maiores prestígios em uma área publicam mais que os com menores prestígios. (VANTI, 2002; SILVEIRA, 2012). (grifo nosso).

Os estudos permitiram chegar à origem da lei dos quadrados inversos, sendo ela caracterizada pela fórmula: "Yx $=6 / \mathrm{p}^{2} \mathrm{xa}$, onde: $Y \mathrm{x}$ é a frequência de autores publicando, $\mathrm{x}=$ número de trabalhos e 'a' é um valor constante para cada campo científico." (SILVA ET AL, 2012, p. 4).

E a Lei de Zipf também conhecida como Lei do Mínimo Esforço determina a relação e a ordem de série das palavras num texto considerado grande, e a palavra com maior constância de ocorrência é classificada com a série 1 e a segunda com a série 2 e assim sucessivamente. (SILVA ET AL, 2012; RIBEIRO, 2013; SILVEIRA, 2012). (grifo nosso).

\subsection{CONGRESSO BRASILEIRO DE CUSTOS}

Segundo Ribeiroet al (2011) quando se trata do assuntos específicos de "custos", o Congresso Brasileiro de Custos é o principal evento do Brasil, sendo ele realizado anualmente.

Este evento permite a interação entre os acadêmicos, pesquisadores, professores, administradores e demais profissionais. O CBC é o principal divulgador da produção técnicocientífica quando relacionada a custos empresariais. (RIBEIRO ET AL, 2011).

O Congresso Brasileiro de Custos e a Associação Brasileira de Custos surgiram em 1994, contudosua origem remonta ao ano de 1987, no primeiro Congresso Internacional de Professores Internacionais de Costos na Argentina, em que os docentes presentes dispuseram criar um Instituto Internacional de Custos e entidades nacionais nos diversos países. (ABC, 2014).

Em 1993, no III Congresso Internacional de Costos, realizado em Madri, Espanha, aprovou-se a proposta do Instituto de Economia da Universidade Estadual de Campinas e do Departamento 
de Contabilidade e Atuária da Universidade de São Paulo para que o IV congresso fosse realizado no Brasil. (ABC, 2014).

Em novembro de 1994, realizou-se, portanto, o I Congresso Brasileiro de Gestão Estratégia de Custos, sendo reunidos docentes universitários do Brasil e profissionais da área, sendo num total de 253 pessoas. Após o congresso, criou-se a Associação Brasileira de Custos (ABC) para que docentes universitários e profissionais pudessem estudar a gestão de custos sob a ótica interdisciplinar. (ABC, 2014).

O CBC tem o objetivo de prover contribuições teóricas para ampliar a literatura acadêmica, com a finalidade de incentivar futuros estudos ou projetos. E no ano de 2014 ocorrerá a $21^{\mathrm{a}}$ edição, sendo realizado na cidade de Natal - Rio Grande do Norte.

O CBC nos três últimos anos teve 7 (sete) temáticas, sendo elas: Metodologias de ensino e pesquisa em custos, Custos como ferramenta para o planejamento, controle e apoio a decisão, Métodos quantitativos aplicados a gestão de custos, Custos aplicados ao setor privado e terceiro setor, Custos aplicados ao setor público, Contribuições teóricas para a determinação e a gestão de custos e Abordagens contemporâneas de custos. (CBC, 2013a).

A primeira é Metodologias de ensino e pesquisa em custos e serve como: "instrumentos, ferramentas e métricas desenvolvidas e/ou adaptadas para facilitar ou melhorar o ensino e a pesquisa na área de custos.". A segunda éCustos como ferramenta para o planejamento, controle e apoio a decisõese tem por finalidade verificar o "uso das metodologias de custeio na controladoria, no processo de planejamento e controle empresarial, na gestão estratégica e no apoio à tomada de decisões.”. (CBC, 2013a, p.1).

A terceira é Métodos quantitativos aplicados à gestão de custos, sendo que fornece a "incorporação de métodos estatísticos, matemáticos e de pesquisa operacional para melhorar a gestão de custos.". A quarta trata de Custos aplicados ao setor privado e terceiro setore aborda "metodologias de custeio desenvolvidas e/ou adaptadas às necessidades de áreas específicas do setor privado e do terceiro setor (agronegócio, para estatais, ONGs, etc.).”. (CBC, 2013a, p.1). A quinta é Custos aplicados ao setor público e aborda "metodologias de custeio desenvolvidas e/ou adaptadas às necessidades do setor público.". A sexta é Contribuições teóricas para a determinação e a gestão de custos e avalia os "avanços teóricos que acrescentem melhorias aos métodos de determinação, mensuração e gestão dos custos.”.(CBC, 2013a, p.1).

E a sétima é Abordagens contemporâneas de custos e elabora "estudos que incorporem tendências na área de custos: custos logísticos, custos da qualidade, custos interorganizacionais, custos intangíveis, custos ambientais, custos de transação, custos da complexidade etc.”. (CBC, 2013a, p.1).

\section{Metodologia}

O presente artigo, em sua metodologia, tem aspectos bibliográficos, documental, descritivo, qualitativa e quantitativa, sendo caracterizado como um estudo bibliométrico.

Muitas pessoas confundem a pesquisa bibliográfica com a documental, entretanto o primeiro é mais vasto que o segundo, mas lembrando, que podem ser realizados concomitantemente. (OLIVEIRA, 1999).

A pesquisa bibliográfica trata do levantamento da bibliografia já publicada sobre determinado tema, principalmente, em livros, revistas, entre outros, (MARCONI; LAKATOS, 2001), sendo, portanto, de fontes secundárias (RODRIGUES, 2006) e contribuicientificamente com o assunto pesquisado. (OLIVEIRA, 1999). 
O objetivo desta pesquisa é colocar o leitor em contato direto com materiais escrito sobre o assunto (MARCONI; LAKATOS, 2001) e permite ao cientista "o reforço paralelo na análise de suas pesquisas ou manipulação de suas informações.”.(TRUJILLO, 1974, p. 230).

A pesquisa documental engloba os materiais que não foram elaborados e que servem como fonte de informação para a pesquisa científica(MARCONI; LAKATOS, 2001), sendo oriundas de fontes primárias, pois os materiais não receberam tratamento analítico. (RODRIGUES, 2006). Os locais mais comuns de se encontrá-los são em arquivos públicos ou particulares e em fontes estatísticas compiladas por órgãos oficiais e particulares. (MARCONI; LAKATOS, 2001).

A pesquisa descritiva aborda aspectos gerais e amplos de um contexto social, sendo que permite desenvolver um nível de análise proporcionando identificar as diferentes formas dos fenômenos. (OLIVEIRA, 1999).

A abordagem qualitativa é classificada por não empregar dados estatísticos como centro da análise do problema, pois não tem pretensão de medir unidades. A pesquisa qualitativa possui como foco situações complexas ou particulares. (OLIVEIRA, 1999), e usando esta abordagem o pesquisador tenta descrever a complexidade de uma situação, analisa a interação de variáveis e interpreta dados, fatos e teorias. (RODRIGUES, 2006).

A abordagem quantitativa quantifica opiniões, dados, nas formas de coleta de informações, com a aplicação da estatística.(RODRIGUES, 2006). Este método é mais usual no desenvolvimento de pesquisas descritivas, com a finalidade de classificar a relação entre as variáveis. (OLIVEIRA, 1999).

As temáticas dos 3 últimos CBC(s)foram: Metodologias de ensino e pesquisa em custos; Custos como ferramenta para o planejamento, controle e apoio a decisão; Métodos quantitativos aplicados a gestão de custos; Custos aplicados ao setor privado e terceiro setor; Custos aplicados ao setor público; Contribuições teóricas para a determinação e a gestão de custos e Abordagens contemporâneas de custos. (CBC, 2013a).

Nestas temáticas foram encontrados 627 artigos. Os dados necessários para a realização do presente trabalho foram coletados no CD-ROM do Congresso Brasileiro de Custos e transportados para o software Excel®

Para a coleta dos dados referentes ao sexo dos autores e das faculdades que eles estão vinculados procedeu a análise da referência sobre estes, que aparecem após o título dos artigos. É precípuo lembrar que no nome dos autores que possuíam mais de uma faculdade, considerou-se apenas a primeira.

$\mathrm{Na}$ parte relacionada ao levantamento da metodologia dosartigos publicados no $\mathrm{CBC}$, inicialmente emanou a leitura dos resumos, sendo que nos artigos que não mencionaram a abordagem no resumo procedeu-se a leitura dos procedimentos metodológicos.

Para análise das palavras-chave, a coleta foi feita no tópico "palavras-chave" dos artigos.

\section{ReSUltados}

Os resultados são apresentados em tabelas para que fique mais fácil a compreensão.

Tabela 1 - Total de artigos

\begin{tabular}{l|c|c|c|c|c|c|c|c}
\hline & MQAGC & ACC & CTDGC & CASPTS & CASP & CFPCAD & MEPC & Total \\
\hline 2011 & 11 & 46 & 11 & 21 & 30 & 57 & 23 & $\mathbf{1 9 9}$ \\
2012 & 19 & 30 & 14 & 22 & 31 & 97 & 22 & $\mathbf{2 3 5}$ \\
2013 & 14 & 36 & 4 & 16 & 23 & 75 & 25 & $\mathbf{1 9 3}$ \\
\hline
\end{tabular}




\begin{tabular}{lcccccccc}
\hline Total & 44 & 112 & 29 & 59 & 84 & 229 & 70 & 627 \\
\hline Fonte: Pesquisa autores & & & & & &
\end{tabular}

Fonte: Pesquisa autores

Em conformidade a tabela 1 verifica que o incondicional de artigos aprovados e publicados nos três últimos CBC(s) nas 7 temáticas foi de 627, deste total 199 no ano de 2011, 235 em 2012 e 193 no ano de 2013. O presente estudo não corrobora aos estudos de Ferrariet al (2012), que encontraram ocorrência de aumento de publicação nas suas pesquisas.

$\mathrm{Na}$ análise por temática verifica que aquela com maior número de artigos publicados é CFPCAD (229), seguida pela ACC (112), CASP (84), MEPC (70), CASPTS (59), MQAGC (44) e CTDGC (29).

Então, verifica que não existe uniformidade na publicação dos artigos nos 3 últimos Congressos Brasileiros de Custos, pois de 2011 para 2012 houve aumento, mas de 2012 para 2013 houve retração. Percebe-se também a predominância de artigos na temática Custos como ferramenta para o planejamento, controle e apoio a decisão.

Verifica que os estudos que buscam aplicar os custos nas entidades empresarias são os que mais possuem publicações, demonstrando assim, que é essencial a ocorrência de aplicação de métodos de custos nas empresas. Logo, o profissional contábil deve buscar a atualização máxima, principalmente no que se refere a custos, pois estudos teóricos existem muitos, mas é preciso aplicar a teoria na prática.

Verifica também que o custo aplicado ao setor público possui boa participação no CBC e logo a sua aplicação na prática é muito importante, principalmente após a publicação da Resolução do CFC n ${ }^{\circ} 1.366 / 11$ que obriga a aplicação de custos na área pública, pois apresenta os sistemas de informações de custos no setor público. (CFC, 2011).

Tabela 2 - Média e Desvio Padrão dos artigos

\begin{tabular}{l|r|l|r|r|r|r|r}
\hline & MQAGC & ACC & CTDGC & CASPTS & CASP & CFPCAD & MEPC \\
\hline Média & 14,67 & 37,33 & 9,67 & 19,67 & 28,00 & 76,33 & 23,33 \\
Desvio Padrão & 3,30 & 6,60 & 4,19 & 2,62 & 3,56 & 16,36 & 1,25 \\
\hline
\end{tabular}

Fonte: Pesquisa autores

A média é a medida de posição mais comum e intuitiva, sendo usada em grandes números de casos práticos. Entretanto, seu uso requer cuidado, devido sofrer influência de todos os valores presentes no intervalo analisado. (MARTINS; DOMINGUES, 2011).No presente trabalho foi usada para verificar qual o número médio de artigos publicados por ano em cada temática nos 3 últimos Congressos Brasileiros de Custos.

Utilizou-se, também o desvio padrão que para Martins e Domingues (2011) é a mais importante medida de dispersão, pois indica dispersão média absoluta dos dados que envolvem a média aritmética.

Os resultados apresentados na tabela 2 demonstram que o melhor resultado encontrado foi na temática MEPC, pois ela tema quarta maior média e possui o menor desvio padrão, demonstrando a maior uniformidade entre as publicações ocorridas nestes anos. Nas demais temáticas existe dispersões maiores, o fator é que não tem uniformidade na quantidade de artigos ao longo do lapso temporal estudado.

Tabela 3-Sexo dos autores

\begin{tabular}{r|r|r|r|l|r|r|r|r}
\hline & \multicolumn{9}{|c|}{ Masculino } & \multicolumn{4}{|c}{ Feminino } \\
\hline & 2011 & 2012 & 2013 & Total & 2011 & 2012 & 2013 & \\
\hline MQAGC & 30 & 44 & 21 & 95 & 8 & 22 & 28 & 58 \\
\hline
\end{tabular}

R. Cont. Ufba, Salvador-Ba, v. 9, n. 1, p. 58 - 72, jan-mar 2015 


\begin{tabular}{lrrrrrrrr}
\hline ACC & 91 & 38 & 52 & 181 & 63 & 57 & 66 & 186 \\
CTDGC & 19 & 23 & 8 & 50 & 11 & 22 & 3 & 36 \\
CASPTS & 46 & 42 & 33 & 121 & 26 & 30 & 23 & 79 \\
CASP & 61 & 56 & 51 & 168 & 37 & 47 & 39 & 123 \\
CFPCAD & 111 & 135 & 146 & 392 & 79 & 180 & 110 & 369 \\
MEPC & 45 & 33 & 43 & 121 & 36 & 41 & 41 & 118 \\
Total & 403 & 371 & 354 & 1128 & 260 & 399 & 310 & 969 \\
\hline
\end{tabular}

Fonte: Pesquisa autores

Em conformidade a tabela 3 verifica que existe predominância de autores dos sexos masculinos (1128) contra 969 do sexo feminino.

Para os autores do sexo masculino verifica que ao longo do lapso temporal existe a predominância de quedas de autoria, pois 2011 foram 403, em 2012371 e em 2013 354, sendo que a presente pesquisa não corrobora com os estudos de Ferrari et al (2012), pois nas pesquisas deles existe crescente considerável de autores do sexo masculino.

Nos autores do sexo feminino verifica que não existe uniformidade, pois os valores encontrados foram 260, 399 e 310, para os anos de 2011, 2012 e 2013, respectivamente.

$\mathrm{Na}$ análise por temáticas verifica que apenas a temática ACC teve predominância de autores do sexo feminino 186 contra 181 do masculino. Então, percebe que na maioria das temáticas tem maior quantidade de autores do sexo masculino.

O presente trabalho corrobora com os estudos de Souza, Silva e Araújo (2012), Ferrari et al (2012) e Santos (2013), que também encontraram predominância de autores do sexo masculino em seus estudos.

Este estudo servirá de alicerce para que as mulheres contabilistas busquem escreverem mais artigos para os congressos, principalmente, o CBC.

Tabela 4 - Autores mais prolíferos

\begin{tabular}{lrrrr|r|r|r|r|r}
\hline & MQAGC & ACC & CTDGC & CASPTS & CASP & CFPCAD & MEPC & Total \\
\hline Alceu Souza & 1 & 1 & 0 & 1 & 0 & 5 & 0 & 8 \\
Altair Borgert & 3 & 0 & 1 & 0 & 1 & 0 & 3 & 8 \\
Carlos E. F. Lavarda & 0 & 2 & 0 & 0 & 0 & 6 & 0 & 8 \\
Fabrícia Silva Rosa & 0 & 4 & 0 & 0 & 0 & 3 & 1 & 8 \\
Ilse Maria Beuren & 0 & 0 & 0 & 0 & 1 & 5 & 2 & 8 \\
Sérgio Murilo Petri & 1 & 1 & 3 & 1 & 0 & 2 & 0 & 8 \\
Total Autores & 153 & 367 & 86 & 200 & 291 & 761 & 239 & 2097 \\
\hline
\end{tabular}

Fonte: Pesquisa autores

Em atenção à tabela 4, verifica que os autores mais prolíferos foram: Alceu Souza, Altair Borgert, Carlos Eduardo Facin Lavarda, Fabrícia Silva da Rosa, Ilse Maria Beuren e Sérgio Murilo Petri, sendo que cada um publicou 8 artigos no Congresso Brasileiro de Custos nos três últimos eventos.

O primeiro autor teve a maior quantidade de artigos publicados na temática CFPCAD com 5 publicações e 1 para MQAGC, ACC e CASPTS. O segundo teve 3trabalhos nas temáticas MQAGC e MEPC e 1 para CTDGC e CASP.

O terceiro autor publicou 6 artigos na temática CFPCAD e 2 em ACC. A quarta autora destacouse em ACC com 4 publicações, 3 para CFPCAD e 1 para MEPC. A quinta autora teve a maior parte dos artigos publicados no CFPCAD com 5 trabalhos, seguido de 2 para MEPC e 1 para CASP. E o sexto autor foi destaque na temática CTDGC com 3 publicações, após CFPCAD 
com 2 trabalhos e com 1 publicação encontra-se as temáticas MQAGC, ACC, CASPTS. Percebe-se que, $66,67 \%$ dos autores mais prolíferos são do sexo masculino.

O presente trabalho corrobora com os estudos de Fariaet al (2013), Ribeiro et al (2011) e Santos (2013)que encontraram entre os autores mais prolíferos Ilse Maria Beuren.Destaca-se que este trabalho também corrobora com os estudos de Suave e Codesso (2012) e Ribeiro et al (2011) que encontraram Carlos Eduardo Facin Lavarda entre os autores mais prolíferos. Este também corrobora com os estudos de Custódio, Machado e Gibbon (2011) e Santos (2013) que tiveram entre os autores mais prolíferos Altair Borget.

Entretanto, não existe corroboração com os estudos de Botinhaet al (2011), Carneiro e Cherobim (2011), Beurenet al (2011), Ribeiro, Silva Sobrinho e Pain (2011), Marques e Santos (2011), Girioli e Asbahr(2013) e Santana, Afonso e Fagundes (2013) que não tiveram nenhum dos autores encontrados neste trabalho como autor mais prolíferos em seus trabalhos.

Tabela 5 - Faculdades mais prolíferas

\begin{tabular}{|c|c|c|c|c|c|c|c|c|}
\hline & MQAGC & ACC & CTDGC & CASPTS & CASP & CFPCAD & MEPC & Total \\
\hline UFSC & 33 & 38 & 13 & 21 & 14 & 57 & 30 & 206 \\
\hline FURB & 10 & 25 & 2 & 18 & 12 & 65 & 20 & 152 \\
\hline UFU & 32 & 20 & 3 & 9 & 3 & 39 & 22 & 128 \\
\hline UFC & 3 & 19 & 0 & 8 & 20 & 35 & 9 & 94 \\
\hline UFPR & 0 & 19 & 1 & 13 & 4 & 30 & 12 & 79 \\
\hline USP & 2 & 12 & 12 & 3 & 17 & 10 & 10 & 66 \\
\hline UFMG & 2 & 9 & 0 & 1 & 17 & 23 & 12 & 64 \\
\hline UEM & 0 & 7 & 0 & 12 & 0 & 26 & 10 & 55 \\
\hline $\begin{array}{l}\text { UNISINOS } \\
\text { Sem }\end{array}$ & 0 & 3 & 7 & 1 & 9 & 28 & 3 & 51 \\
\hline Vínculo & 2 & 5 & 1 & 4 & 6 & 20 & 0 & 38 \\
\hline UFSM & 5 & 6 & 0 & 1 & 0 & 25 & 0 & 37 \\
\hline UnB & 3 & 4 & 0 & 0 & 11 & 14 & 3 & 35 \\
\hline Total & 153 & 367 & 86 & 200 & 291 & 761 & 239 & 2097 \\
\hline
\end{tabular}

Fonte: Pesquisa autores

Com os dados apresentados na tabela 5, verifica-se que a faculdade mais prolífera foi a Universidade Federal de Santa Catarina (UFSC) com um total de 206 publicações nos 3 últimos CBC(s), sendo que a temática mais prolífera foi CFPACD com 57 vezes. Destaca-se, também os autores que não estão vinculados a nenhuma universidade que totalizaram 38 aparições, isto demonstra que o CBC atinge a profissionais que não estão ligados a nenhuma universidade.

A presente pesquisa corrobora com os estudos de Melo et al (2012a) e Silva et al (2012) que encontraram a Universidade Federal de Santa Catarina (UFSC) como a mais prolífera.

Por outro lado, o presente estudo diverge dosestudos de Lima, Diniz e Silva (2012) e de Santana, Afonso e Fagundes (2013) que encontraram como faculdade mais prolífera a UFMG. Os trabalhos de Freitas (2012), Marques e Santos (2011), Machado, Silva e Beuren (2011) e Santos (2013) que encontram que como faculdade mais prolífera a USP. 
Tabela 6 - Abordagem mais usual

\begin{tabular}{l|ccccc|cccccc|c|c|c|c|ccc|c}
\hline & \multicolumn{4}{|c|}{ Quantitativo } & \multicolumn{4}{c|}{ Qualitativo } & \multicolumn{4}{c|}{ Quant./Qualitativo } & \multicolumn{4}{c}{ Não mencionado } \\
\hline & 11 & 12 & 13 & Total & 11 & 12 & 13 & Total & 11 & 12 & 13 & Total & 11 & 12 & 13 & Total \\
\hline MQAGC & 6 & 9 & 3 & 18 & 1 & 1 & 1 & 3 & 1 & 3 & 1 & 5 & 3 & 6 & 9 & 18 \\
ACC & 12 & 3 & 6 & 21 & 16 & 11 & 4 & 31 & 5 & 8 & 2 & 15 & 13 & 8 & 24 & 45 \\
CTDGC & 1 & 1 & 1 & 3 & 2 & 5 & & 7 & 3 & 3 & & 6 & 5 & 5 & 3 & 13 \\
CASPTS & 5 & 7 & 5 & 17 & 7 & 6 & 1 & 14 & 3 & 4 & 1 & 8 & 6 & 5 & 9 & 20 \\
CASP & 14 & 5 & 7 & 26 & 5 & 12 & 1 & 18 & 2 & 10 & 3 & 15 & 9 & 4 & 12 & 25 \\
CFPCAD & 14 & 27 & 13 & 54 & 17 & 31 & 19 & 67 & 13 & 12 & 4 & 29 & 13 & 27 & 39 & 79 \\
MEPC & 8 & 10 & 4 & 22 & 8 & 3 & 3 & 14 & 6 & 6 & 8 & 20 & 1 & 3 & 10 & 14 \\
Total & 6 & 62 & 39 & 161 & 56 & 69 & 29 & 154 & 33 & 46 & 19 & 98 & 50 & 58 & 106 & 214 \\
\hline
\end{tabular}

Fonte: Pesquisa autores

Em conformidade a tabela 6, verifica que dos 627 artigos publicados no lapso temporal, 214 não tiveram classificação, quanto à abordagem da metodologia utilizada pelos autores. Após apareceu os estudos com pesquisa quantitativa que apresentaram 161 trabalhos, os com pesquisa qualitativa tiveram 154 artigos e a abordagem quantitativa/qualitativafoi encontrada em 98 artigos.

O presente estudo corrobora com os estudos de Ferrari et al (2012), pois os autores encontraram nos seus estudos que os artigos por eles estudados, que não tiveram abordagem definida foi o mais usual.

Entretanto, a presente pesquisa não corrobora com os estudos de Melo et al (2012b),Lima, Diniz e Silva (2012), Carneiro e Cherobim (2011), Santana, Afonso e Fagundes (2013) e Oliveira et al (2013) que encontraram que a pesquisa mais usual é a qualitativa.E com os estudos de Oliveira e Aragão (2011) e Pereira e Moura (2011) apresentaram que a abordagem mais prolífera foi aqualitativa e quantitativa.

Os pesquisadores devem focar mais em apresentar a abordagem que usaram no artigo, pois está é fundamental para o bom entendimento do trabalho.

Tabela 7 - Palavras-chave mais prolíferas

\begin{tabular}{l|c|c|c|c|c|c|c|c|c|c|c|c|c|c|c|c|c|c|c|c|c|c}
\hline & \multicolumn{4}{|c|}{$\begin{array}{c}\text { Uma palavra- } \\
\text { chave }\end{array}$} & \multicolumn{4}{c|}{$\begin{array}{c}\text { Duas palavras- } \\
\text { chave }\end{array}$} & \multicolumn{4}{c|}{$\begin{array}{c}\text { Quatro palavras- } \\
\text { Thês palavras-chave }\end{array}$} & \multicolumn{4}{c}{$\begin{array}{c}\text { Cinco palavras- } \\
\text { chave }\end{array}$} \\
\hline & 11 & 12 & 13 & Total & 11 & 12 & 13 & Total & 11 & 12 & 13 & Total & 11 & 12 & 13 & Total & 11 & 12 & 13 & Total \\
\hline MQAGC & 0 & 0 & 0 & 0 & 0 & 0 & 1 & 1 & 11 & 13 & 13 & 37 & 0 & 4 & 0 & 4 & 1 & 2 & 0 & 3 \\
ACC & 0 & 0 & 0 & 0 & 0 & 0 & 0 & 0 & 41 & 27 & 28 & 96 & 3 & 3 & 6 & 12 & 2 & 0 & 2 & 4 \\
CTDGC & 0 & 0 & 0 & 0 & 0 & 0 & 0 & 0 & 10 & 9 & 4 & 23 & 1 & 3 & 0 & 4 & 0 & 2 & 0 & 2 \\
CASPTS & 1 & 0 & 0 & 1 & 0 & 0 & 1 & 1 & 19 & 17 & 14 & 50 & 0 & 4 & 1 & 5 & 1 & 1 & 0 & 2 \\
CASP & 0 & 0 & 0 & 0 & 0 & 1 & 1 & 2 & 25 & 26 & 16 & 67 & 2 & 4 & 6 & 12 & 3 & 0 & 0 & 3 \\
CFPCAD & 1 & 0 & 0 & 1 & 2 & 1 & 0 & 3 & 50 & 78 & 60 & 189 & 4 & 15 & 13 & 32 & 0 & 3 & 1 & 4 \\
MEPC & 0 & 0 & 0 & 0 & 0 & 0 & 0 & 0 & 22 & 20 & 20 & 62 & 0 & 2 & 3 & 5 & 1 & 0 & 2 & 3 \\
Total & $\mathbf{2}$ & $\mathbf{0}$ & $\mathbf{0}$ & $\mathbf{2}$ & $\mathbf{2}$ & $\mathbf{2}$ & $\mathbf{3}$ & $\mathbf{7}$ & $\mathbf{1 7 8}$ & $\mathbf{1 9 0}$ & $\mathbf{1 5 5}$ & $\mathbf{5 2 3}$ & $\mathbf{1 0}$ & $\mathbf{3 5}$ & $\mathbf{2 9}$ & $\mathbf{7 4}$ & $\mathbf{8}$ & $\mathbf{8}$ & $\mathbf{5}$ & $\mathbf{2 1}$ \\
\hline
\end{tabular}

Fonte: Pesquisa autores 
O presente estudo demonstra, mediante a tabela 7, que o maior número de palavras-chave utilizadas nos artigos publicados pelo CBC(s) nos três últimos anos foi de 3 palavras-chave, que totalizou 523 trabalhos, representando uma percentagem de 83,41\%. Após, apareceu os artigos que possuem 4 palavras-chave, sendo que tiveram um total de 74 trabalhos, depois encontra-se que os trabalhos com 5 palavras-chave apresentam um total de 21 artigos, verifica que com 2 palavras-chave teve 7 artigos e com 1 palavra-chave encontrou 2 artigos.

O modelo de artigo do Congresso Brasileiro de Custos recomenda que se usem três palavraschave (CONGRESSO BRASILEIRO DE CUSTOS, 2013b), mas não determina a obrigatoriedade desta quantidade. Com os resultados encontrados na presente pesquisa percebe que a maioria dos autores utilizam a quantidade de palavras-chave que o $\mathrm{CBC}$ recomenda.

\section{CONSIDERAÇÕES FINAIS}

O presente artigo teve por objetivo analisar o perfil dos artigos publicados no Congresso Brasileiro de Custos nos 3 últimos anos. Os principais pontos avaliados foram os números de artigos publicados, o gênero de autores predominante, os autores com mais trabalhos publicados, as instituições mais prolíferas, a abordagem mais usual e o maior número de palavras-chave utilizadas nos artigos.

As temáticas analisadas foram: Metodologias de ensino e pesquisa em custos (MEPC), Custos como ferramenta para o planejamento, controle e apoio a decisão (CFPCAD), Métodos quantitativos aplicados a gestão de custos (MQAGC), Custos aplicados ao setor privado e terceiro setor (CASPTS), Custos aplicados ao setor público (CASP), Contribuições teóricas para a determinação e a gestão de custos (CTDGC) e Abordagens contemporâneas de custos (ACC). (CBC, 2013a).

Os resultados encontrados demonstraram que o número de artigos publicados ao longo do lapso temporal não é uniforme, pois existe aumento do primeiro para o segundo ano e queda do segundo para o terceiro, e que na aplicação da média e do desvio padrão o melhor resultado foi verificado na MEPC.

Constatou-se que há predominância de autores do sexo masculino em relação ao feminino, sendo que apenas na temática ACC houve predominância de autores do sexo feminino em comparação ao masculino. Encontrou que os autores mais prolíferos foram: Alceu Souza, Altair Borgert, Carlos Eduardo Facin Lavarda, Fabrícia Silva da Rosa, Ilse Maria Beuren e Sérgio Murilo Petri, sendo que cada um teve 8 trabalhos aprovados para apresentação e publicação nos anais do Congresso Brasileiro de Custos nos três últimos anos.

Verificou que a faculdade mais prolífera foi a UFSC, que teve 206 publicações ao longo do evento, e que teve como temática mais prolífera a CFPACD, com 57 vezes.

No trato da abordagem verificou que na maioria dos artigos os autores não mencionaram qual abordagem que eles usaram em seus artigos.

Verificou, quanto ao número de palavras-chave, que o mais usual são 3, sendo presente em $83,41 \%$ dos trabalhos e seguindo a recomendação do modelo de artigo do CBC.

O presente trabalho apresentou como limitações: o lapso temporal pequeno, apenas 3 anos e trabalhar apenas com um congresso.

Para futuras pesquisas recomenda-se a análise de mais congressos, o que permite realizar a comparação entre congressos, com a finalidade de verificar uma similaridade. Pode também aplicar modelos estáticos específicos para verificar as principais redes entre os autores.

As temáticas do CBC são importantes, pois abordam 4 diferentes áreas de aplicação dos custos, mas é importante que o congresso busque uma temática com foco exclusivo nas mudanças pela qual a Contabilidade vem passando, com o processo de convergência. 


\section{REFERÊNCIAS}

ASSOCIAÇÃO Brasileira de Custos - ABC. Apresentação. 2014. Disponível em: <http://cbc2011.edugraf.ufsc.br/?page_id=41> Acesso em: 12 jan. 2014.

CONSELHO Federal de Contabilidade. Resolução CFC nº 1.366/11 - Aprova a NBC T 16.11 - Sistema de Informação de Custos do Setor. Diário Oficial da União, Brasília, DF, 12 dez. 2011. Seção 1.

CONGRESSO Brasileiro de Custos - CBC, 2013a. Áreas temáticas. Disponível em:<http://cbc.edugraf.ufsc.br/?page_id=48>. 2013. Acesso em: 3 jan. 2014.

CONGRESSO Brasileiro de Custos - CBC, 2013b. Disponível em:

$<$ http://cbc2013.emnuvens.com.br/wp-

content/uploads/2009/05/modelo_artigo_custos_20131.doc>. Acesso em: 30 dez. 2013

BEUREN, Ilse Maria et al. Métodos de Custeio: Uma Meta-Análise dos Artigos Publicados no Congresso Brasileiro de Custos no Período de 1994 a 2010. In: CONGRESSO

BRASILEIRO DE CUSTOS, 18., 2011, Rio de Janeiro. Anais.... São Leopoldo: Associação Brasileira de Custos, 2011.

BOTINHA, Reiner Alves et al. Gestão de riscos: levantamento da produção científica em eventos nacionais de contabilidade e administração entre 2008 e 2010. In: CONGRESSO BRASILEIRO DE CUSTOS, 18., 2011, Rio de Janeiro. Anais.... São Leopoldo: Associação Brasileira de Custos, 2011.

CARNEIRO, Leandro Marcondes; CHEROBIM, Ana Paula MussiSzabo. Teoria de agência em sociedades cooperativas: estudo bibliométrico a partirda produção científica nacional. In: CONGRESSO BRASILEIRO DE CUSTOS, 18., 2011, Rio de Janeiro. Anais.... São Leopoldo: Associação Brasileira de Custos, 2011.

CUSTÓDIO, Eduardo Barbosa; MACHADO, Débora Gomes; GIBBON, Artur Roberto de Oliveira. Custeio Baseado em Atividades: Análise da Produção Científica, sob a perspectiva bibliométrica e sociométrica, do Congresso Brasileiro de Custos,no Período de 2006 a 2010. In: CONGRESSO BRASILEIRO DE CUSTOS, 18., 2011, Rio de Janeiro. Anais.... São Leopoldo: Associação Brasileira de Custos, 2011.

FARIA, Paula MiekoOda et al. Pesquisa em Contabilidade Gerencial no Brasil: um estudo bibliométrico de 2002 a 2012 nos principais periódicos nacionais. In: CONGRESSO BRASILEIRO DE CUSTOS, 20., 2013, Uberlândia. Anais.... São Leopoldo: Associação Brasileira de Custos, 2013.

FERRARI, Patrícia da Silva et al. Análise da produção científica do Congresso Brasileiro de Custos, na área de Ensino e Pesquisa em contabilidade no período de 1989a 2009. In: CONGRESSO BRASILEIRO DE CUSTOS, 19., 2012, Gramado. Anais.... São Leopoldo: Associação Brasileira de Custos, 2012. 
FREITAS, Claudio Luiz de. Custos ambientais: área temática ou publicações 'isoladas'? Uma análise das publicações do Congresso Brasileiro de Custos sob a ótica da contribuição dos pesquisadores para o desenvolvimento da temática. In: CONGRESSO BRASILEIRO DE CUSTOS, 19., 2012, Gramado. Anais.... São Leopoldo: Associação Brasileira de Custos, 2012.

GIRIOLI, Lumila Souza; ASBAHR, Daiane de F. Análise da produção científica sobre Custeio Baseado em Atividades aplicado ao setor de serviços no Congresso Brasileiro de Custos entre 1994 e 2012. In: CONGRESSO BRASILEIRO DE CUSTOS, 20., 2013, Uberlândia. Anais.... São Leopoldo: Associação Brasileira de Custos, 2013.

LIMA, Francielly Dornelas Correia; DINIZ, Jéssica Rosa; SILVA, Denise Mendes.Perfil de Produção Científica em Contabilidade: um comparativoentre os periódicos Contabilidade Vista \& Revista e UniversoContábil no período de 2006 a 2010. In: CONGRESSO BRASILEIRO DE CUSTOS, 19., 2012, Gramado. Anais.... São Leopoldo: Associação Brasileira de Custos, 2012.

LAROCCA, Priscila; ROSSO, Ademir José; SOUZA, Audrey Pietrobelli. A formulação dos objetivos de pesquisa napós-graduação: uma discussão necessária. Revista Brasileira de PósGraduação - Capes,Brasília, v. 1, n. 1, p. 118-133, 2005.

MACHADO, Débora Gomes; SILVA, Tarcísio Pedro da; BEUREN, Ilse Maria. Produção Científica de Custos: Análise das Publicações em Periódicos Nacionais de Contabilidade sob a perspectiva das Redes Sociais e da Bibliometria. In: CONGRESSO BRASILEIRO DE CUSTOS, 18., 2011, Rio de Janeiro. Anais.... São Leopoldo: Associação Brasileira de Custos, 2011.

MARCONI, Marina de Andrade; LAKATOS, Eva Maria. Metodologia do Trabalho

Científico: Procedimentos básicos, pesquisa bibliográfica, projeto e relatório e publicações e trabalhos científicos. 6. ed. São Paulo: Atlas, 2001.

MARQUES, Leandro; SANTOS, Vanderlei dos. Abordagem dinâmica do capital de giro em trabalhos publicados nos periódicos nacionais Qualis CAPES. In: CONGRESSO

BRASILEIRO DE CUSTOS, 18., 2011, Rio de Janeiro. Anais.... São Leopoldo: Associação Brasileira de Custos, 2011.

MARTINS, Gilberto de Andrade; DOMINGUES, Osmar. Estatística Geral e Aplicada. 4. ed. revisada e ampliada. São Paulo: Altas, 2011.

MATURANA, Humberto R; VARELA Francisco G. A árvore do conhecimento: as bases biológicas do entendimento humano. Campinas: Psy, 1995. p.p. 240.

MELO, Diego Vieira et al.Contabilidade e Custos Ambientais: Um Mapeamento das Produções Científicas em Periódicos e Eventos Nacionais. In: CONGRESSO BRASILEIRO DE CUSTOS, 19., 2012a, Gramado. Anais.... São Leopoldo: Associação Brasileira de Custos, 2012.

MELO, Diego Vieira et al. Contabilidade e Custos Ambientais: Um Mapeamento dasProduções Científicas em Periódicos e Eventos Nacionais. In: CONGRESSO 
BRASILEIRO DE CUSTOS, 19., 2012b, Gramado. Anais.... São Leopoldo: Associação Brasileira de Custos, 2012.

MONTEIRO, Jonas Eduardo Rocha et al. Ética e contabilidade: estudo bibliométrico das publicações dosanais do Enanpad na primeira década do Século XXI. In: XIV SemeAD Seminários emAdministração, 14., 2011, Ceará. Anais.... São Paulo: Universidade Federal de São Paulo, USP, 2011.

MORAES, Romildo de Oliveira. Gestão Estratégica de Custos: Investigação da Produção Científica no Período de 2008 a 2012. In: CONGRESSO BRASILEIRO DE CUSTOS, 20., 2013, Uberlândia. Anais.... São Leopoldo: Associação Brasileira de Custos, 2013.

OLIVEIRA, Ana Caroline Peixoto; ARAGÃO, Iracema Raimunda Brito Neves. Perfil das pesquisas em Contabilidade de Custos publicadas nas revistason-line avaliadas pela Coordenação de Aperfeiçoamento de Pessoal de NívelSuperior. In: CONGRESSO BRASILEIRO DE CUSTOS, 18., 2011, Rio de Janeiro. Anais.... São Leopoldo: Associação Brasileira de Custos, 2011.

OLIVEIRA, Silvio Luiz de. Tratado de Metodologia Científica: projetos de pesquisas, TGI, TCC, Monografias, Dissertações e Teses. São Paulo: Pioneira, 1999.

OLIVEIRA, Sheila Cristina Macário et al. Bibliometria em artigos de contabilidade aplicada ao setor público.In: CONGRESSO BRASILEIRO DE CUSTOS, 20., 2013, Uberlândia. Anais.... São Leopoldo: Associação Brasileira de Custos, 2013.

PEREIRA, Nevison Amorim; MOURA, Marcelino Franco. Custos no agronegócio: um estudo bibliométrico dos anos de 2003a 2013.In: CONGRESSO BRASILEIRO DE CUSTOS, 20., 2013, Uberlândia. Anais.... São Leopoldo: Associação Brasileira de Custos, 2013.

RIBEIRO, Silvio Paula; SILVA SOBRINHO, Priscila; PAIN, Juliano Aparecido. Estudo Bibliométrico da Produção Científica do CBC de 2004-2010 na Sessão Novas Tendências Aplicadas na Gestão de Custos. In: CONGRESSO BRASILEIRO DE CUSTOS, 18., 2011, Rio de Janeiro. Anais.... São Leopoldo: Associação Brasileira de Custos, 2011.

RIBEIRO, Silvio Paula et al. Estudo bibliométrico em controladoria no congresso brasileiro de custos no período de 2004 a 2010. In: CONGRESSO BRASILEIRO DE CUSTOS, 18., 2011, Rio de Janeiro. Anais.... São Leopoldo: Associação Brasileira de Custos, 2011.

RIBEIRO, Henrique César Melo. Abordagem da Governança Corporativa em artigos publicados nas revistas da área de Contabilidade classificadas no sistema Qualis da Capes de 2000 a 2011. Revista Brasileira de Contabilidade. Brasília, n. 199, p. 11-27, jan./fev. 2013.

RODRIGUES, Auro de Jesus. Metodologia Científica: complexo e essencial para a vida do universitário. São Paulo: Avercamp, 2006.

SANTANA, Alex Fabiano Bertollo; AFONSO, Paulo; FAGUNDES, Jair Antônio. Time DrivenActivityBasedCosting (TDABC): Um perfil dos artigos publicados no Congresso Brasileiro de Custos.In: CONGRESSO BRASILEIRO DE CUSTOS, 20., 2013, Uberlândia. Anais.... São Leopoldo: Associação Brasileira de Custos, 2013. 
SANTOS, Ananias Francisco do. Análise Bibliométrica da produção científica sobre custos nos principais periódicos contábeis no Brasil no século XXI. In: CONGRESSO BRASILEIRO DE CUSTOS, 20., 2013, Uberlândia. Anais.... São Leopoldo: Associação Brasileira de Custos, 2013.

SILVA, Ana Paula Ferreira da et al. Estudo bibliométrico sobre custo em organizações da construção civil: contribuições do congresso brasileiro de custo de 1996 a2010. In: CONGRESSO BRASILEIRO DE CUSTOS, 19., 2012, Gramado. Anais.... São Leopoldo: Associação Brasileira de Custos, 2012.

SILVEIRA, João Paulo Borges. A produção científica em periódicos institucionais: um estudo da Revista Biblos. Revista eletrônica de biblioteconomia e ciência da informação. Pelotas, v. 17, n. 33, p. 116-133, jan./abr., 2012.

SLOMSKI, Vilma Geni et al. A importância da formulação da questão de pesquisa na produção científica em contabilidade: uma discussão a partir de trabalhos publicados no Congresso Brasileiro de Custos no ano de 2009. In: CONGRESSO BRASILEIRO DE CUSTOS, 20., 2013, Uberlândia. Anais.... São Leopoldo: Associação Brasileira de Custos, 2013.

SOUZA, Fábia Jaiany Viana de; SILVA, Maurício Corrêa da; ARAÚJO, Aneide Oliveira. Uma análise da produção científica da área de contabilidade governamental nos periódicos que utilizam o SEER e o SciELO. In: Congresso USP de Iniciação Científica em Contabilidade, 9., 2012, São Paulo. Anais eletrônicos... Disponível em: <http://www.congressousp.fipecafi.org/artigos122012/35.pdf>. Acesso em: 01 jan. 2014.

SUAVE, Ricardo; CODESSO, Maurício Mello. Produção científica sobre orçamento: análise das principais características de revistas brasileiras. In: CONGRESSO BRASILEIRO DE CUSTOS, 19., 2012, Gramado. Anais.... São Leopoldo: Associação Brasileira de Custos, 2012.

TRUJILLO, Afonso Ferrari. Metodologia da ciência. 3. ed. Rio de Janeiro: Kennedy, 1974.

VANTI, Nadia Aurora Peres. Da bibliometria à webometria: uma exploração conceitualdos mecanismos utilizados para medir o registro da informação e a difusão doconhecimento.

Ciência da Informação, Brasília, v. 31, n. 2, p. 369-379, maio/ago. 2002.

VIAUD, Gaston. A inteligência: a sua evolução e as suas formas. Lisboa: Publicações Europa-América, 1964. 